\title{
Retour sur « Des Idées pour observer »
}

\section{François Sigaut}

\section{(2) OpenEdition}

Journals

Édition électronique

URL : https://journals.openedition.org/tc/5046

DOI : $10.4000 /$ tc. 5046

ISSN : 1952-420X

\section{Éditeur}

Éditions de l'EHESS

\section{Édition imprimée}

Date de publication : 30 juin 2010

Pagination : 84-86

ISSN : 0248-6016

\section{Référence électronique}

François Sigaut, « Retour sur « Des Idées pour observer » », Techniques \& Culture [En ligne], 54-55 |

2010, mis en ligne le 30 janvier 2013, consulté le 29 septembre 2022. URL : http://

journals.openedition.org/tc/5046 ; DOI : https://doi.org/10.4000/tc.5046 


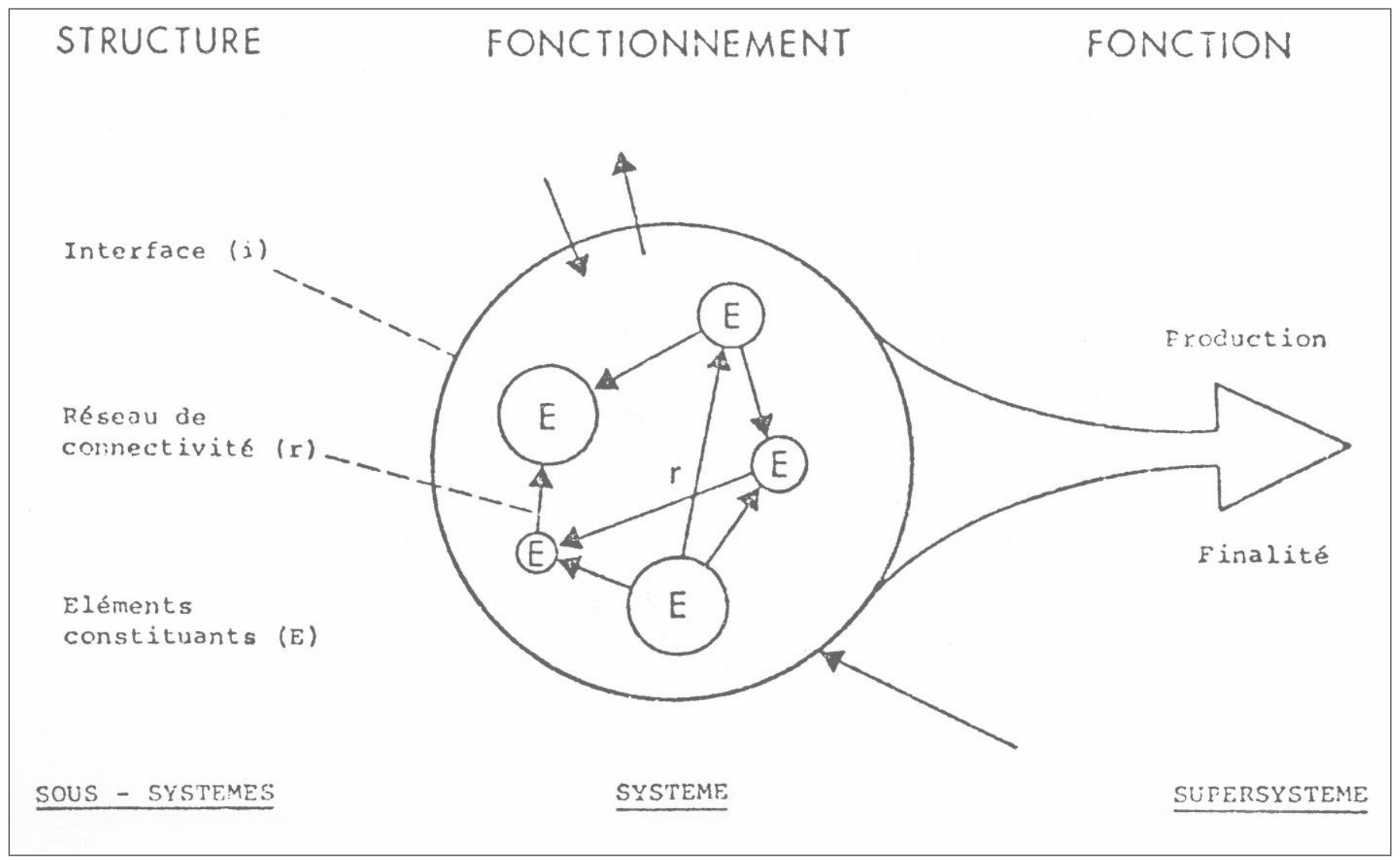

Fig. 1. - Schéma général d'organisation d'un integron comme unité oystémique.

La spécification du système par la structure distingue : l'inferface (i), frontière morphologique et lieu d€s Łchanges entre le système et son environnement, les composants élémentaires (E) ou sous-systèmes, et le réseau de relation $(r)$ qui solidarise ces éléments.

La spécification du système par son fonctionnement suppose la description de la nature et de la dynamique des relations " fonctionnelles * qui unissent les divers sous-systimes qui le composent.

La fonclion du système est considérée comme le produit de son fonctionnement. La finalité de cette fonction s'exprime dans le réseau de relations fonctionnelles du supersystème qui intègre le système considéré comme un de ses éléments. 


\section{Retour sur}

\section{"Des Idées pour observer »}

En relisant cet petit texte, j'ai eu d'abord un regret : celui de n’avoir pas cité Jacques Paillard, neurophysiologiste, qui avait publié en 1976 un article tout à fait fondamental sur les concepts de forme/fonctionnement/fonction ${ }^{1}$. Il n'était d'ailleurs pas le premier à aborder ce sujet. On en trouve une discussion plus ou moins élaborée chez J.-L. Maunoury en 1968, J. Piaget en 1967, L. Couffignal en 1963...2 Maunoury était économiste, Piaget psychologue et Couffignal cybernéticien : c'est dire combien la question transcende les disciplines. Elle concerne toutes les approches en termes de système, Paillard le montre excellemment. Mais ce n'est pas en fréquentant ces diverses œuvres que j'ai été amené à m'y intéresser, ce serait plutôt l'inverse. Au départ, il s'agissait pour moi d'un problème fort précis. À quoi sert une charrue ? À labourer, répond-on d'ordinaire. Oui mais... à quoi sert de labourer? C'est là que gît le lièvre. Une charrue, un araire étant donnés, et dont on ne sait rien par ailleurs, on peut essayer de reconstituer expérimentalement le travail qu'ils permettent d'effectuer, au moins en principe. Mais à supposer qu'on y parvienne (l'archéologie expérimentale est un terrain semé de pièges), on n’aura répondu qu'à la moitié de la question. Pourquoi en effet ce travail-là et pas un autre ? Il ne suffit pas de se poser la question de ce que fait l'instrument, il faut encore se poser celle de savoir pourquoi il fait ceci plutôt que cela. C'est-à-dire que pour comprendre son fonctionnement, il faut savoir quelque chose de sa fonction. C'est le seul moyen d'arriver à comprendre pourquoi il y a tant de modèles différents d'instruments aratoires.

L'exemple des instruments aratoires n'est peut-être pas le meilleur, du reste, d'un point de vue pédagogique. Je crois que celui des marteaux le vaut bien. Non pas parce que nous nous y connaissons mieux en marteaux qu'en charrues, mais parce que, me semble-t-il, nous avons tous eu un jour l'occasion de nos étonner de la forme bizarre de tel ou tel modèle. Or il est bien clair, ici, que chaque modèle de marteau doit les spécificités 
de sa forme aux détails des gestes en usage dans le métier qui l'emploie. Nous avons beau ne pas connaître ces gestes, voire ne pas nous y intéresser, nous comprenons très bien, au vu d'une collection de marteaux, que c'est en nous interrogeant sur eux que nous trouverions la solution - c'est presque instinctif! Et qu'est-ce qui permet de rendre compte de ces gestes (qui sont des fonctionnements) ? Les besoins du métier, c'est-à-dire les fonctions qui en définissent l'exercice.

Cela me fait penser à ce précepte si souvent répété, qui est de « replacer les choses dans leur contexte». C'est fort bien, mais où commence ce fameux contexte, et surtout où finit-il ? Si on part à l'aventure, on risque de s'égarer dans un « contexte» qui peut être tout et n'importe quoi, et de passer ainsi à côté de l'essentiel. Il y faut un peu de méthode. Un peu, pas trop, parce que les méthodologies trop rigides sont toujours stérilisantes. Mais un peu tout de même, et c'est ce minimum que le schéma forme/fonctionnement/ fonction représente à mon sens assez bien. Un schéma dont le domaine de validité dépasse de loin la seule Technologie, comme l'avaient vu J. Paillard et ses émules.

C'est ainsi que, m'interrogeant sur la répartition des activités entre hommes et femmes (un thème aussi ancien que l'anthropologie), j'ai été amené à constater que dans d'assez nombreux cas, l'esclavage avait eu une fonction importante, qui était d'imposer à des hommes des tâches normalement féminines (la mouture des grains par exemple) et parfois l'inverse (les femmes guerrières). Pourquoi, dans la masse immense des écrits sur l'esclavage, cette fonction apparaît-elle si peu? Je ne suis pas tout à fait sûr de la réponse, mais il me semble tout de même que s'il en est ainsi, c'est parce que la plupart des auteurs se sont interrogés sur les fonctionnements de l'esclavage, pas sur ses fonctions proprement dites. Peut-être parce que celles-ci leur paraissaient aller de soi. Et il est vrai que ce fut le cas de l'esclavage colonial, dont les fonctions furent presque exclusivement économiques. Mais quelle qu'ait été l'importance de l'esclavage colonial, on ne peut pas en faire un modèle universel. Avant le XIX ${ }^{e}$ siècle, la plupart des sociétés qui avaient les moyens d'acquérir des esclaves, en avaient, et la diversité des fonctions de l'esclavage était considérable.

Rien n'est plus simple, finalement, que la distinction entre fonctionnement et fonction. Peut-être même est-ce parce qu'elle est si simple que dans les sciences sociales où on a plutôt le goût de la complexité, on en a fait aussi peu de cas. Ce serait alors une des forces de la technologie, science humaine que d'offrir un domaine dans lequel la simplicité pourrait s'avérer particulièrement féconde. 